POS PROCEEDINGS

\title{
NLO QCD Production of Higgs plus jets with GoSam
}

\section{G. Cullen}

Deutsches Elektronen-Synchrotron DESY, Platanenallee 6, 15738 Zeuthen, Germany

E-mail: gavin.cullen@desy.de

H. van Deurzen, N. Greiner, G. Heinrich, G. Luisoni, E. Mirabella, T. Peraro, J. Reichel, J. Schlenk, J.F. von Soden-Fraunhofen

Max Planck Institute for Physics, Föhringer Ring 6, 80805 Munich, Germany

E-mail: \{hdeurzen, greiner, gudrun, luisonig, mirabell, peraro, joscha, jschlenk, jfsoden\}@mpp.mpg.de

\section{P. Mastrolia}

Max Planck Institute for Physics, Föhringer Ring 6, 80805 Munich, Germany;

Dipartimento di Fisica e Astronomia, Università di Padova, and INFN Sezione di Padova, via Marzolo 8, 35131 Padova, Italy

E-mail: ppaolo@mpp.mpg.de

\section{G. Ossola*}

Physics Department, New York City College of Technology, The City University of New York, 300 Jay Street Brooklyn, NY 11201, USA;

The Graduate School and University Center, The City University of New York, 365 Fifth Avenue, New York, NY 10016, USA

E-mail: gossola@citytech. cuny.edu

\section{F. Tramontano}

Dipartimento di Scienze Fisiche, Università degli studi di Napoli and INFN, Sezione di Napoli, 80125 Napoli, Italy

E-mail: francesco.tramontanodcern.ch

\begin{abstract}
After reviewing the main features of the GOSAM framework for automated one-loop calculations, we present a selection of recent phenomenological results obtained with it. In particular, we focus on the recent calculation of NLO QCD corrections to the production of a Higgs boson in conjunction with jets at the LHC.
\end{abstract}

The European Physical Society Conference on High Energy Physics

18-24 July, 2013

Stockholm, Sweden

\footnotetext{
*Speaker.
} 


\section{Introduction}

The large amount of data accumulated by the experimental collaborations at the Large Hadron Collider (LHC) allowed for a very detailed investigation of the Standard Model (SM) of particle physics. Moreover, the discovery of a Higgs boson with mass of about $126 \mathrm{GeV}$ [1] finally confirmed the validity of the electroweak symmetry breaking mechanism [2].

In all these analyses, for example to further study the properties of the recently discovered Higgs boson, theory predictions play a fundamental role. They are not only needed for the signal, but also for the modeling of the relevant background processes, which share similar experimental signatures. Further, precise theory predictions are important in order to constrain model parameters in the event that a signal of New Physics is detected. Since leading-order (LO) results are affected by large uncertainties, theory predictions are not reliable without accounting for higher orders. Therefore, it is of primary interest to provide theoretical tools which are able to perform the comparison of LHC data to theory at NLO accuracy.

In the past few years, the progress in the automation of NLO calculations for multi-particle final states has been tremendous and led to the so-called "NLO revolution" [3]. Several automated frameworks for one-loop calculations [4] have been presented, which are based on various new theoretical developments [6]. It is fascinating to witness the number and quality of advanced automated NLO calculations that have been performed with different techniques.

In this presentation, we review the main features of the GoSAM framework [5] for the automated computation of one-loop amplitudes. GoSAM has been recently employed in several calculations at NLO QCD accuracy [7-14] related to signal and backgrounds for Higgs boson production, as well as in the context of Beyond Standard Model (BSM) scenarios $[15,16]$ and electroweak studies [17], and has been successfully interfaced with Monte Carlo programs to merge multiple NLO matrix elements with parton showers $[18,19]$.

We also briefly describe a selection of recent phenomenological results obtained with GoSAM, with particular attention to the recent calculations of NLO QCD corrections to the production of Higgs boson in conjunction with jets at the LHC.

\section{The GoSAM framework}

GoS AM combines automated diagram generation and algebraic manipulation [20-23] with integrand-level reduction techniques [24]. Amplitudes are generated via Feynman diagrams, using QGRAF [20], FORM [21], spinney [23] and haggies [22]. The individual program tasks are managed by python scripts, so that the only task required from the user is the preparation of an input file in order to launch the generation of the source code and its compiling, without having to worry about the internal details. The input file contains specific information about: i) the process, such as a list of initial and final state particles, the order in the coupling constants, and the model; ii) the scheme employed, such as the regularization and renormalization schemes; iii) the system, such as paths to libraries or compiler options; iv) optional information to control the code generation.

After the generation of all contributing diagrams, the virtual corrections are evaluated using the $d$-dimensional integrand-level reduction method, as implemented in SAMURAI [26] library, which allows for the combined determination of both cut-constructible and rational terms at once. 
Alternatively, the tensorial decomposition provided by GoLEM95C [27-29] is also available. Such reduction, which is numerically stable but more time consuming, is employed as a rescue system. After the reduction, all relevant master integrals can be computed by means of GoLEM95C [29], QCDLOOP [30], or ONELOOP [31].

As a novel approach to the integrand reduction, the method proposed in [25] allows for the extraction of all the coefficients in the integrand decomposition by performing a Laurent expansion, whenever the analytic form of the numerator function is known. This method has been implemented, within the GoSAM framework, in the C++ library NINJA, showing an improvement in the computational performance, both in terms of speed and precision, with respect to the standard algorithms. More details are provided in the talk of T. Peraro at this conference [32]. The new library has been recently employed in the evaluation of NLO QCD corrections to $p p \rightarrow t \bar{t} H j$ [13].

GoSAM can be used to generate and evaluate one-loop corrections in both QCD and electroweak theory. Model files for BSM theories can be generated from a Universal FeynRules Output (UFO) [33] or LanHEP [34] file.

Code development New features have been recently implemented within GoSAM, with respect to the current public version. In order to deal with the complexity level of calculations such as $p p \rightarrow H j j j$ [12], the GoSAM code has been enhanced. On the one side, the generation algorithm has been improved by a more efficient diagrammatic layout: Feynman diagrams are grouped according to their topologies, namely global numerators are constructed by combining diagrams that have a common set, or subset, of denominators, irrespectively of the specific particle content. On the other side, additional improvements in the performances of GoSAM have been achieved by exploiting the optimized manipulation of polynomial expressions available in FORM 4.0 [35]. The possibility of employing numerical polarization vectors and the option to sum diagrams sharing the same propagators algebraically during the generation of the code led to an enormous gain in code generation time and reduction of code size.

Concerning the amplitude reduction, aside from the already mentioned new integrand-level reduction via Laurent expansion [25], GoSAM has been enhanced to reduce integrands that may exhibit numerators with rank larger than the number of the denominators. This is indeed the case in the presence of effective couplings [9,12], which appear in the large top-mass approximation, or when dealing with spin-2 particles [16]. For these cases, within the context of integrand-reduction techniques, the parametrization of the residues at the multiple-cut has to be extended and the decomposition of any one-loop amplitude acquires new master integrals [25]. The extended integrand decomposition has been implemented in the SAMURAI library [36].

The new developments regarding the improved generation and reduction algorithms will be publicly available in the GOSAM 2.0 release, which is currently in preparation.

Interfacing with MC and BLHA The computation of physical observables at NLO accuracy, such as cross sections and differential distributions, requires to combine the one-loop results for the virtual amplitudes obtained with GoSAM, with other tools that can take care of the computation of the real emission contributions and of the subtraction terms, needed to control the cancellation of IR singularities. 
This can be obtained by embedding the calculation of virtual corrections within a Monte Carlo framework (MC), that can take care of the phase-space integration, and of the combination of the different pieces of the calculation. A table with a comprehensive list of GoSAM interfaces with MC programs has been recently presented in [37].

In order to facilitate the communication between the programs computing virtual one-loop amplitudes and the MC frameworks, a standard interface called the Binoth Les Houches Accord (BLHA) [38] has been designed. Within the BLHA, the interaction between the One-loop Program (OLP) and the Monte Carlo framework (MC) proceeds in two phases. During the first phase, called pre-runtime phase, the MC creates an order file, which contains information about the setup and the subprocesses needed from the OLP in order to perform the computation. The OLP reads the order file, checks availability for each item, and returns a contract file telling the MC what it can provide. In the second stage, the MC requires from the OLP the values of the virtual one-loop amplitudes at specific phase-space points.

\section{Higgs boson production in Gluon Fusion}

At the LHC, the dominant Higgs production mechanism proceeds via gluon fusion (GF), where the coupling of the Higgs to the gluons is mediated by a heavy quark loop. For this reason, the calculation of higher order corrections for the GF production of a Higgs boson in association with jets has received a lot of attention in the theory community over the past decade [39].

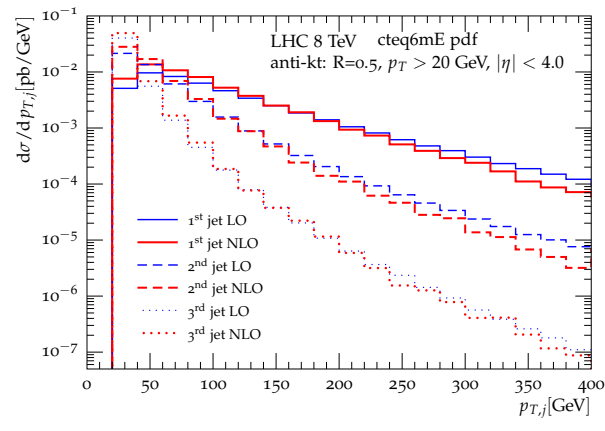

(a)

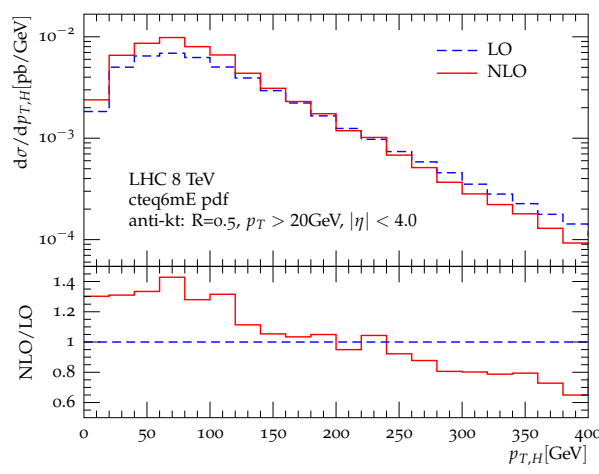

(b)

Figure 1: $p p \rightarrow H+3 j$ in GF for the LHC at $8 \mathrm{TeV}$ : (a) transverse momentum distributions of the leading jets, (b) transverse momentum distribution of the Higgs boson.

The developments in GoSAM, described in Section 2, allowed to compute the NLO QCD corrections to the production of $H+2$ jets [9] and, for the first time, also $H+3$ jets [12] in GF (in the large top-mass limit). While a fully automated BLHA interface between GoSAM and SHERPA [40] has been used for $p p \rightarrow H+2 j$, the complexity of the integration for the process $p p \rightarrow H+3 j$ forced us to employ a hybrid setup which combines GoSAM, SHERPA and the MadDipole/Madgraph4/MadEvent framework [41]. This calculation is indeed challenging both on the side of real-emission contributions and of the virtual corrections, which alone involve more than 10,000 one-loop Feynman diagrams with up to rank-seven hexagons.

In the calculation of $H+3$ jets the cteq $6 \mathrm{~L} 1$ and cteq $6 \mathrm{mE}$ parton-distribution functions were used for LO and NLO respectively, and a minimal set of cuts based on the anti- $k_{T}$ jet algorithm 
with $R=0.5, p_{T, \min }>20 \mathrm{GeV}$ and $|\eta|<4.0$ was applied. Figure 1 shows the $p_{T}$ distributions of the three jets and of the Higgs boson, respectively. The NLO corrections enhance all distributions for $p_{T}$ values lower than $150-200 \mathrm{GeV}$, whereas their contribution is negative at higher $p_{T}$.

This study also shows that the virtual contributions for $p p \rightarrow H j j j$ generated by GoSAM is ready to be paired with available Monte Carlo programs to aim at further phenomenological studies.

\section{Other Phenomenological results}

Diphotons+jets GOSAM in combination with MadDipole/MadGraph4/MadEvent has been used to calculate the NLO QCD corrections to $p p \rightarrow \gamma \gamma+1$ jet [10] and $p p \rightarrow \gamma \gamma+2$ jets [11], where the former also includes the fragmentation component. This calculation allowed for a first reliable prediction of the absolute normalization of this process, and demonstrated that the shape of important kinematical distributions is modified by higher-order effects.

Beyond the Standard Model GoSAM has been used to calculate the NLO Susy-QCD corrections to the production of a pair of the lightest neutralinos plus one jet at the $\mathrm{LHC}$ at $8 \mathrm{TeV}$, which appears as a monojet signature in combination with missing energy. All non-resonant diagrams have been fully included, namely without using the assumption that production and decay factorize. We observe that the NLO corrections to the missing transverse energy are large, mainly due to additional channels opening up at NLO. The detailed setup can be found in [15].

Another recent BSM result obtained with GoSAM+MadDipole/MadGraph4/MadEvent is the calculation of NLO QCD corrections to the production of a graviton in association with one jet [16], where the graviton decays into a photon pair, within ADD models of large extra dimensions [42]. The calculation is quite complicated due to the tensor structure introduced by spin-2 particles, and the non-standard propagator of the graviton, coming from the summation over Kaluza-Klein modes. It is interesting to notice that the $K$-factors of the invariant mass distribution of the photon pair emitted in the decay of the graviton are not uniform. Since the latter is used to derive exclusion limits, it is advisable to take into account the effect of NLO corrections. For details we refer to [16].

Higgs+Vector Boson+jet GOSAM was interfaced with the POWHEG BOX to compute the associate production of a Higgs boson, a vector boson, and one jet [18]. In this calculation, the improved MiNLO procedure [43] was used to obtain NLO accurate predictions also when the jet is not resolved. Using this interface the generation of any process is fully automated, except for the construction of the Born phase space.

NLO QCD corrections to $p p \rightarrow t \bar{t} H j$ The production rate for a Higgs boson associated with a top-antitop pair $(t \bar{t} H)$ is particularly interesting to study the properties of the newly discovered Higgs boson, since it is directly proportional to the SM Yukawa coupling of the Higgs boson to the top quark. We recently presented the complete NLO QCD corrections to the process $p p \rightarrow t \bar{t} H+1$ jet $(t \bar{t} H j)$ at the LHC [13]. The goal of the calculation was twofold. On the one hand, it is important for the phenomenological analyses at the LHC, in particular for the high- $p_{T}$ region, where the presence of the additional jet can be relevant. On the other hand, from the technical point of view, for the presence of two mass scales (Higgs boson and top quark) and internal massive particles, together with a high number of diagrams, $p p \rightarrow t \bar{t} H j$ constitutes a challenge for many reduction 
algorithms. This calculation represents the first application of the novel reduction algorithm, implemented in the library NINJA, based on integrand-level reduction via Laurent expansion [25].

\section{Conclusions}

GoSAM is a flexible and widely applicable tool for the automated calculation of the virtual part of multi-particle scattering amplitudes at NLO accuracy. After interfacing it with MC programs, that can perform integration over phase space and combine the contributions coming from real emission and subtraction terms as well, total cross-sections and differential distributions can be easily obtained for a variety of processes of interest at the LHC.

Boosted by state-of-the-art techniques for the reduction of the scattering amplitudes, GoSAM provides a reliable answer for multi-leg amplitudes in the presence of massive internal and external legs and propagators, such as the production of a Higgs boson in conjunction with a top-quark pair, as well as in configurations with relatively high multiplicity, such as Higgs boson plus jets production. While the GoSAM code will be further improved, it will be interesting to observe whether the attempt of extending integrand-level techniques to higher orders [44] will succeed and provide a comparable level of automation, at least for the calculation of the virtual parts.

Other challenges for the near future involve interfacing GOSAM with MC programs for an automated generation of the full cross section including parton showering, and ultimately the production of codes and results to be used within experimental analyses. We believe that the amount of recent calculations that were produced with the GoSAM framework shows, both in terms of stability and precision, that it is an ideal multi-purpose tool for studying the physics of the LHC.

\section{Acknowledgments}

The work of G.C. was supported by DFG Sonderforschungsbereich Transregio 9, Computergestützte Theoretische Teilchenphysik and the support of the Research Executive Agency (REA) of the European Union under the Grant Agreement number PITN-GA-2010-264564 (LHCPhenoNet). H.v.D., G.L., P.M., and T.P. are supported by the Alexander von Humboldt Foundation, in the framework of the Sofja Kovaleskaja Award Project "Advanced Mathematical Methods for Particle Physics", endowed by the German Federal Ministry of Education and Research. The work of G.O. was supported in part by the National Science Foundation under Grant PHY-1068550 and PSC-CUNY Award No. 65188-00 43. This research work benefited of computing resources from the Rechenzentrum Garching and the the CTP cluster of the New York City College of Technology.

\section{References}

[1] Aad G et al. (ATLAS Collaboration) 2012 Phys.Lett. $B 716$ 1-29; Chatrchyan S et al. (CMS Collaboration) 2012 Phys.Lett. B716 30-61

[2] Englert F and Brout R 1964 Phys.Rev.Lett. 13 321-323; Higgs P W 1964 Phys.Lett. 12 132-133

[3] Salam G P 2010 PoS ICHEP2010 556; Perret-Gallix D 2013 J.Phys.Conf.Ser. 454012051

[4] Berger C, Bern Z, Dixon L, Febres Cordero F, Forde D et al. 2008 Phys.Rev. D78 036003; Bevilacqua G, Czakon M, Garzelli M, van Hameren A, Kardos A et al. 2013 Comput.Phys.Commun. 184 986-997; Hirschi V, Frederix R, Frixione S, Garzelli M V, Maltoni F et al. 2011 JHEP 1105 044; 
Cascioli F, Maierhofer P and Pozzorini S 2012 Phys.Rev.Lett. 108 111601; Agrawal S, Hahn T and Mirabella E 2012 PoS LL2012 046; Badger S, Biedermann B, Uwer P and Yundin V 2013 Comput.Phys.Commun. 184 1981-1998; Actis S, Denner A, Hofer L, Scharf A and Uccirati S 2013 JHEP 1304037

[5] Cullen G, Greiner N, Heinrich G, Luisoni G, Mastrolia P et al. 2012 Eur.Phys.J. C72 1889

[6] Ellis R K, Kunszt Z, Melnikov K and Zanderighi G 2012 Phys.Rept. 518 141-250; Bern Z, Dixon L and Kosower D 2007 Annals Phys. 322 1587; Ossola G 2013 (Preprint 1310 . 3214)

[7] Greiner N, Guffanti A, Reiter T and Reuter J 2011 Phys.Rev.Lett. 107102002

[8] Greiner N, Heinrich G, Mastrolia P, Ossola G, Reiter T et al. 2012 Phys.Lett. B713 277-283

[9] van Deurzen H, Greiner N, Luisoni G, Mastrolia P, Mirabella E et al. 2013 Phys.Lett. B721 74-81

[10] Gehrmann T, Greiner N and Heinrich G 2013 JHEP 1306058

[11] Gehrmann T, Greiner N and Heinrich G 2013 (Preprint 1308 . 3660)

[12] Cullen G, van Deurzen H, Greiner N, Luisoni G, Mastrolia P et al. 2013 Phys.Rev.Lett. 111131801

[13] van Deurzen H, Luisoni G, Mastrolia P, Mirabella E, Ossola G et al. 2013 (Preprint 1307 . 8437)

[14] Dolan M J, Englert C, Greiner N and Spannowsky M 2013 (Preprint 1310.1084 )

[15] Cullen G, Greiner N and Heinrich G 2013 Eur.Phys.J. C73 2388

[16] Greiner N, Heinrich G, Reichel J and von Soden-Fraunhofen J F 2013 (Preprint 1308 . 2194)

[17] Chiesa M, Montagna G, Barze‘ L, Moretti M, Nicrosini O et al. 2013 Phys.Rev.Lett. 111 121801; Mishra K, Becher T, Barze L, Chiesa M, Dittmaier S et al. 2013 (Preprint 1308.1430)

[18] Luisoni G, Nason P, Oleari C and Tramontano F 2013 (Preprint 1306 . 2542)

[19] Hoeche S, Huang J, Luisoni G, Schoenherr M and Winter J 2013 Phys.Rev. D88 014040

[20] Nogueira P 1993 J.Comput.Phys. 105 279-289

[21] Vermaseren J A M 2000 (Preprint math-ph/0010025)

[22] Reiter T 2010 Comput.Phys.Commun. 181 1301-1331

[23] Cullen G, Koch-Janusz M and Reiter T 2011 Comput.Phys.Commun. 182 2368-2387

[24] Ossola G, Papadopoulos C G and Pittau R 2007 Nucl.Phys. B763 147-169; Ossola G, Papadopoulos C G and Pittau R 2007 JHEP 0707 085; Ellis R K, Giele W T and Kunszt Z 2008 JHEP 03 003; Ossola G, Papadopoulos C G and Pittau R 2008 JHEP 0805 004; Mastrolia P, Ossola G, Papadopoulos C and Pittau R 2008 JHEP 0806030

[25] Mastrolia P, Mirabella E and Peraro T 2012 JHEP 1206095

[26] Mastrolia P, Ossola G, Reiter T and Tramontano F 2010 JHEP 1008080

[27] Binoth T, Guillet J P, Heinrich G, Pilon E and Reiter T 2009 Comput.Phys.Commun. 180 2317-2330

[28] Heinrich G, Ossola G, Reiter T and Tramontano F 2010 JHEP 1010105

[29] Cullen G, Guillet J, Heinrich G, Kleinschmidt T, Pilon E et al. 2011 Comput.Phys.Commun. 182 2276-2284

[30] van Oldenborgh G 1991 Comput.Phys.Commun. 66 1-15; Ellis R K and Zanderighi G 2008 JHEP 02 002 
[31] van Hameren A 2011 Comput.Phys.Commun. 182 2427-2438

[32] Peraro T, presentation at EPS-HEP 2013, in these proceedings

[33] Christensen N D and Duhr C 2009 Comput. Phys. Commun. 180 1614-1641; Degrande C, Duhr C, Fuks B, Grellscheid D, Mattelaer O et al. 2011 (Preprint 1108.2040); Alloul A, Christensen N D, Degrande C, Duhr C and Fuks B 2013 (Preprint 1310.1921)

[34] Semenov A 2010 (Preprint 1005.1909)

[35] Kuipers J, Ueda T, Vermaseren J and Vollinga J 2013 Comput.Phys.Commun. 184 1453-1467

[36] Mastrolia P, Mirabella E, Ossola G, Peraro T and van Deurzen H 2012 PoS LL2012 028 (Preprint 1209.5678)

[37] Cullen G, van Deurzen H, Greiner N, Heinrich G, Luisoni G et al. 2013 (Preprint 1309. 3741)

[38] Binoth T, Boudjema F, Dissertori G, Lazopoulos A, Denner A et al. 2010 Comput.Phys.Commun. 181 1612-1622; Alioli S, Badger S, Bellm J, Biedermann B, Boudjema F et al. 2013 (Preprint $1308.3462)$

[39] Dittmaier S et al. (LHC Higgs Cross Section Working Group) 2011 (Preprint 1101.0593); Dittmaier S, Dittmaier S, Mariotti C, Passarino G, Tanaka R et al. 2012 (Preprint 1201.3084); Heinemeyer S et al. (The LHC Higgs Cross Section Working Group) 2013 (Preprint 1307.1347)

[40] Gleisberg T, Hoeche S, Krauss F, Schonherr M, Schumann S et al. 2009 JHEP 0902007

[41] Frederix R, Gehrmann T and Greiner N 2008 JHEP 0809 122; Frederix R, Gehrmann T and Greiner N 2010 JHEP 1006 086; Stelzer T and Long W 1994 Comput.Phys.Commun. 81 357-371; Maltoni F and Stelzer T 2003 JHEP 0302 027; Alwall J, Demin P, de Visscher S, Frederix R, Herquet M et al. 2007 JHEP 0709028

[42] Arkani-Hamed N, Dimopoulos S and Dvali G 1998 Phys.Lett. B429 263-272; Antoniadis I, Arkani-Hamed N, Dimopoulos S and Dvali G 1998 Phys.Lett. B436 257-263

[43] Hamilton K, Nason P, Oleari C and Zanderighi G 2013 JHEP 1305082

[44] Mastrolia P and Ossola G 2011 JHEP 1111014 (Preprint 1107. 60 41); Badger S, Frellesvig H and Zhang Y 2012 JHEP 1204 055; Zhang Y 2012 JHEP 1209 042; Mastrolia P, Mirabella E, Ossola G and Peraro T 2012 Phys.Lett. B718 173-177; Feng B and Huang R 2013 JHEP 1302 117; Mastrolia P, Mirabella E, Ossola G and Peraro T 2013 Phys.Rev. D87 085026; Mastrolia P, Mirabella E, Ossola G and Peraro T 2013 (Preprint 1307 . 5832); Badger S, Frellesvig H and Zhang Y 2013 (Preprint 1310.1051) 